\title{
TUTORIA COM AGENTES INTELIGENTES NA EDUCAÇÃO ONLINE
}

\author{
Aníbal Guerreiro \\ Daniela Melaré Vieira Barros \\ Lina Morgado ${ }^{*}$
}

\section{INTRODUÇÃO}

Historicamente, o tutor está presente em diversos contextos educacionais, presenciais e online e consiste numa pessoa envolvida na gestão da informação e em outras funções. Nestas incumbências, também designadas de "tutoria, o tutor observa as dificuldades dos estudantes, identifica os aspetos pedagógicos necessários para facilitar a aprendizagem e presta assistência de forma mais célere, eficaz e imediata. Atualmente, a função de tutoria constitui-se também num novo perfil de atuação, mais direto, aperfeiçoado e personalizado, envolvendo várias dimensões para além da pedagógica: a gerencial, a técnica e a social.

A evolução tecnológica permitiu ir além dos ambientes virtuais de aprendizagem, onde estes funcionam como suporte e gestão de conteúdos, e através dos tutores, interpretes dos objetivos do curso, é possível utilizar outras formas de fazer chegar o conhecimento aos alunos. É reconhecido pela literatura da área que os tutores, ao longo do tempo, têm vindo a ganhar importância na EaD, assumindo tarefas essenciais no processo de ensino e aprendizagem.

As qualidades humanas que, em geral, os tutores devem possuir são a empatia, a sociabilidade, a responsabilidade e a capacidade de aceitação; as qualidades científicas incidem no conhecimento didático-pedagógico; e as qualidades técnicas sobre como trabalhar com eficácia, em equipa e o domínio da literacia tecnológica (MORGADO, 2001; 2003; BELLONI 2008; MACHADO \& MACHADO, 2004).

Essas qualidades estão imbuídas de uma construção pedagógica e comunicacional em contextos online, onde o movimento de interação e participação dos elementos envolvidos nos processos de ensino e aprendizagem sejam constantemente acompanhados com interações muitas vezes imediatas. Essa necessidade constante e crescente da personalização e das intervenções diretas e mais rápidas necessita de complementos que potencializam as capacidades e características de uma gestão tutorial.

\footnotetext{
${ }^{(*)}$ Aníbal Guerreiro. Universidade Aberta, Portugal. Orcid: 〈http://orcid.org/0000-0001-6512-6788>.

Daniela M. Barros. Universidade Aberta, Portugal. Orcid: http://orcid.org/0000-0002-1412-2231.

Lina Morgado. Universidade Aberta, Portugal. Orcid: < http://orcid.org/0000-0002-4973-6727.
} 
As tendências de personalização para a educação podem ser consultadas em documentos como o NMC Horizon Report - da "The New Media Consortium", uma entidade focada em discutir novas tendências de média, comunicação e educação. No relatório global, os dados apresentados recentemente sobre o ensino superior apontam, de entre as várias tendências, os ambientes de aprendizagem personalizados e as tecnologias para a aprendizagem adaptativa. Nesta assertiva considera-se a personalização utilizando o processo de tutoria como elemento estratégico de trabalho educativo em contextos online (BARROS ,2018).

De forma indicativa, simples e abrangente, as funções, habilidades e perfil do tutor para formação online estão apresentadas na tabela seguinte:

Tabela 1. Funções, habilidades e perfil do Tutor Online.

\begin{tabular}{|c|c|c|c|}
\hline Função & Competência & Habilidade & Perfil \\
\hline $\begin{array}{l}\text { Ter uma cultura tecnológica } \\
\text { para facilitar a sua } \\
\text { comunicação e a interface } \\
\text { com os alunos. }\end{array}$ & $\begin{array}{l}\text { Em cultura } \\
\text { tecnológica. }\end{array}$ & $\begin{array}{l}\text { Uso de aparelhos digitais, } \\
\text { comunicar-se pelas tecnologias } \\
\text { digitais com os alunos, domínio de } \\
\text { uso dos aparelhos da tecnologia em } \\
\text { geral, compreensão da lógica de } \\
\text { uso dos aparelhos digitais. }\end{array}$ & $\begin{array}{l}\text { Domínio da } \\
\text { informática. }\end{array}$ \\
\hline $\begin{array}{l}\text { Ter o domínio do } \\
\text { computador e compreensão } \\
\text { geral do seu funcionamento. }\end{array}$ & $\begin{array}{l}\text { No uso dos } \\
\text { aplicativos básicos } \\
\text { do computador. }\end{array}$ & $\begin{array}{l}\text { Uso avançado dos aplicativos do } \\
\text { Word, Excel e Power Point, } \\
\text { capacidade de conectar os } \\
\text { periféricos do computador e } \\
\text { resolver pequenos problemas } \\
\text { técnicos. }\end{array}$ & $\begin{array}{l}\text { Domínio da } \\
\text { informática. }\end{array}$ \\
\hline $\begin{array}{l}\text { Estabelecer um espaço com o } \\
\text { docente responsável pela } \\
\text { disciplina, para a troca de } \\
\text { informações pedagógicas e as } \\
\text { dificuldades que } \\
\text { possivelmente poderão ser } \\
\text { sanadas no processo de } \\
\text { ensino-aprendizagem. }\end{array}$ & Na área pedagógica. & $\begin{array}{l}\text { Troca de informações e procura de } \\
\text { informações necessárias sobre os } \\
\text { temas. }\end{array}$ & $\begin{array}{l}\text { Disponibilidade } \\
\text { Responsabilidade. }\end{array}$ \\
\hline $\begin{array}{l}\text { Ter consciência dos aspetos } \\
\text { éticos que envolvem a sua } \\
\text { função em relação aos alunos } \\
\text { e ao docente do curso. }\end{array}$ & Ética e moral. & $\begin{array}{l}\text { Análise e julgamento baseados em } \\
\text { princípios morais e éticos que } \\
\text { norteiam toda e qualquer prática na } \\
\text { vida em geral e não somente na } \\
\text { parte profissional. }\end{array}$ & Responsabilidade. \\
\hline $\begin{array}{l}\text { Atualizar-se constantemente } \\
\text { na área à qual pertence. }\end{array}$ & $\begin{array}{l}\text { Em iniciativa na } \\
\text { procura da formação } \\
\text { continuada. }\end{array}$ & $\begin{array}{l}\text { Organização da vida pessoal, } \\
\text { profissional e académica, para que } \\
\text { a formação continuada seja } \\
\text { constante e natural. }\end{array}$ & $\begin{array}{l}\text { Independência } \\
\text { Disponibilidade } \\
\text { Iniciativa } \\
\text { Organização } \\
\text { Ser estudioso. }\end{array}$ \\
\hline
\end{tabular}




\begin{abstract}
Exercer o papel de motivador do aluno, que por problemas diversos e pessoais pode apresentar dificuldades no cumprimento das atividades propostas.
\end{abstract}

Acompanhar os alunos, auxiliando nas dúvidas académicas, burocráticas e gerais do curso ou disciplina ao qual está vinculado.

Esclarecer dúvidas quanto ao conteúdo da disciplina ou curso, enviando, se necessário, material complementar.

Argumentar, deacordo com os questionamentos, dúvidas e inferências, com responsabilidade, dentro dos prazos propostos.

Procurar antecipar-se às necessidades dos alunos, a partir das características pessoais de cada um.

Encaminhar dúvidas ou questões e procurar ajuda nos momentos necessários, compartilhando informação e conhecimento.

Saber organizar o tempo de acesso ao ambiente virtual de ensino, de modo a desenvolver as atividades com qualidade.

$\begin{array}{lll}\text { Em motivar fazendo } & \text { Escrever de forma empática e } & \text { Capacidade de } \\ \text { uso das palavras. } & \text { motivadora. } & \text { inferência } \\ & & \text { Criatividade } \\ & \text { Empatia. }\end{array}$

Em conhecimento dos processos de gestão do curso em todas as suas vertentes.

$\mathrm{Na}$ área ou conteúdo do curso.

Em capacidade
argumentativa.

Responder e estabelecer um processo de diálogo com os alunos, cumprindo com os compromissos assumidos de forma responsável.

Em relacionamento interpessoal.

Perceber as necessidades do outro de maneira empática, facilitando a resolução do problema de forma criativa.

Em trabalho multi e interdisciplinar

Trabalhar em equipa de maneira colaborativa.

Priorizar tarefas importantes e

Em organização temporal.
Conhecimento de toda a estrutura administrativa e legislativa do curso.

Organização didática do conteúdo, e focando, apresenta dificuldades.
Disponibilidade
Organização
Ser estudioso
Curioso e
argumentativo.

Responsabilidade

Empatia

Criatividade

Disponibilidade

Ser tolerante e ao mesmo tempo exigente.

Responsabilidade

Organização

Independência

Disponibilidade

Iniciativa.

Empatia

Criatividade.

Responsabilidade Disponibilidade Iniciativa Flexibilidade Ser Curioso e Argumentativo. Organização Criatividade Independência Disponibilidade Iniciativa.

Fonte: Adaptado de MORGADO (2001);NASCIMENTO, L. ET AL. (2015); BARROS (2011); BARROS, \& REI, (2009); GONÇALVES, M. H. (2008); CEJUDO, M. (2006).

A tabela supra apresentada, sumariza, de uma forma clara, algumas das principais funções, habilidades e o perfil do tutor, fazendo a respetiva associação e mostrando a relação existente entre elas. Neste cenário, existe a necessidade de as Instituições disporem de tutores multifacetados de forma contínua, interativa, personalizada e à conveniência dos alunos. Para além disso, com acesso regular aos conteúdos, ao feedback e ao apoio nas suas áreas de estudo, juntando, ainda, o que podemos pontuar como vantagens de um agente inteligente ou tutor virtual. Todo esse cenário exige a discussão das novas possibilidades da tutoria, o seu desenvolvimento e evolução.

Deixamos claro neste trabalho que os tutores virtuais são considerados como um apoio complementar ao trabalho da tutoria, serão potencializadores do processo, ampliando as 
capacidades de rapidez e atendimento mais detalhado e personalizado dos estudantes, de acordo com objetivos e competências a serem desenvolvidas. A integração de tutores virtuais, em colaboração com os seus atores (o tutor), procura capturar a riqueza e dinamismo do comportamento humano e, consequentemente, modelar a razão, inteligência emocional, inteligência social, processos fisiológicos, processos cognitivos e expressão (MELO, 2007).

\section{Procedimentos Metodológicos}

A metodologia utilizada neste artigo foi baseada na sistematização das pesquisas e estudos experienciados dentro de um projeto científico financiado e desenvolvido recentemente, juntamente com a investigação exploratória realizada para o desenvolvimento do estado da arte sobre o tema. $\mathrm{O}$ método dedutivo, a partir do processo de pesquisa bibliográfica e documental, conduziu às reflexões e análises dos temas aqui tratados. A construção teórica apresentada tem como base o desenvolvimento do estado da arte sobre a tutoria virtual e os agentes inteligentes.

As opções metodológicas basearam-se numa significativa diversidade de estratégias de recolha de informação, privilegiando o recurso das metodologias qualitativas, tendo em conta o objeto do estudo. Devido à dimensão do trabalho, que limita a introdução de elementos de informação complementares, que poderiam conduzir a processos de triangulação de dados, assim como uma maior contextualização dos estudos, optou-se por designá-lo apenas referenciais que sustentam o estado da arte em desenvolvimento.

A realização do estudo aqui descrito teve como sustentação teórica as referências da tutoria virtual, através dos autores Morgado (2015; 2001), Nascimento, L. et al.(2015), Alves et al., (2003), Amarilla Filho (2011), Sangrá, Vlachopoulos \& Bravo (2011), com Belloni (2008) e Barros \& Reis (2009). Em relação aos agentes inteligentes, as evidências apontadas pelos autores Baylor \& Kim (2005), Rickel, et al. (2002a,b), Perikos \& Hatzilygeroudis (2014), Muldowney (2017), Gomes (2017), Zumstein \& Hundertmark (2017), Srdanovic (2017) e Schappo (2017), permitiram abordar diferentes aspetos e compreender o potencial do seu uso.

\section{AGENTES INTELIGENTES}

No seu ritual comunicativo, os humanos, no seu dia a dia, englobam um elevado número de elementos complexos, como a fala, a gesticulação, o movimento dos olhos e da cabeça, ou a combinação de alguns deles, o que reflete a sua emoção e define o seu comportamento. Simultaneamente, a sociedade vive associada às tecnologias, nomeadamente à conectividade e, nesse sentido, há também um sonho de imitar o comportamento humano, interpretando, 
processando e simulando as suas emoções de forma a poder conferir uma melhor comunicação entre o Homem e a Máquina (JOHNSON, AT. AL. 2015).

No ensino superior online, a presença de um tutor virtual com determinadas características complementa e facilita o trabalho de interação e mediação. $\mathrm{O}$ tutor pode reagir às indicações faciais de um aluno, que de acordo com Mahmoud \& Robinson (2011), podem ser de empatia, alegria, tristeza, surpresa, desilusão, interesse, aborrecimento, reflexão, incerteza, entre outros estados emotivos.

Os agentes computacionais que englobem fatores afetivos/emotivos na sua interação com os seres humanos através da linguagem natural, são referidos por alguns autores como Agentes Virtuais Encapsulados (ECAs) (Hsu, 2012), (Melo, 2007). Esses agentes antropomórficos ou encapsulados procuram capturar a riqueza e dinamismo do comportamento humano e, consequentemente, modelar a razão, inteligência emocional, inteligência social, processos fisiológicos, processos cognitivos e de expressão.

O esforço é multidisciplinar, dado que o que envolve a criação abrange uma panóplia de especialidades, que vão desde as Tecnologias, Desenho Gráfico, Human Computer Interface e outras áreas, como a Sociologia, a Psicologia e Animação Gráfica, (Melo, 2007) refere-se ainda que um agente antropomórfico pode abranger as seguintes áreas de utilização: Visão, Aplicações, Expressão Corporal, Expressão Facial, Expressão Vocal, Expressão Emocional e Controlo de Expressão.

Rickel et al. (2002a) acrescentam que além de ser necessária a colaboração de uma equipa multidisciplinar para o desenvolvimento de um Agente, deve incluir especialistas nas seguintes áreas de investigação: Animação da Figura Humana; Perceção; Modelação Cognitiva, Psicologia Cognitiva; Processamento da Linguagem Natural; Reconhecimento da Fala e Síntese; Comunicação Não Verbal; Simulação Distribuída e Jogos de Computador.

As escolhas relativas àaparência, personalidade e comportamentos dos Agentes são frequentemente feitas com base numa análise introspetiva e de preferências pessoais Rickel, et.al.(2002b). Um agente afetivo que simule um professor humano para ser usado como tutor no ensino online traz grandes benefícios e aumenta o interesse dos estudantes pela aprendizagem CINTO ET AL. ( 2013), CHAO ET. AL. (2012) E LIN E TSAI (2011).

Os investigadores tentam alcançar a eficácia da comunicação natural face-a-face dos seres humanos e, através de um sistema, interagir naturalmente com eles e, assim, compreender e utilizar os mesmos meios de comunicação através de sistemas antropomórficos, quer sob a forma de 
personagens virtuais usando sistemas avançados de computação gráfica em 3D ou na forma de robôs humanóides físicos BECKER-ASANO (2008).

De acordo com Cassel et al. (2000), os Embodied Conversational Agents são personagens cartoonlike, gerados por computador, que demonstram muitas das mesmas propriedades que os seres humanos em conversa face-a-face, incluindo a capacidade de produzir e responder à comunicação verbal e não verbal. Os Agentes constituem um tipo de a) interface multimodal, que é aquela que utiliza a conversação humana: a fala, expressões faciais, gestos e postura corporal; b) agente de software, que representa a interação do humano com o computador ou representam os seus utilizadores "humanos" num ambiente computacional (como por exemplo: avatares); e c) sistema de diálogo, onde ambos os dispositivos, verbais e não verbais, regulam o diálogo entre o utilizador e o computador.

Os denominados "embodied agents" são um caso particular de agentes inteligentes que têm forma física ou virtual. Um “agent conversational” é um agente que tem a capacidade de conversar. Da combinação e evolução de ambos surgiram os Embodied Conversational Agents (ECA), que resultam da transposição da riqueza interativa entre humanos para a interação entre humanos e máquinas MARSI, ROODEN (2007).

Baylor (2009) defende que os agentes virtuais antropomórficos podem servir de mediadores tecnológicos para acrescentar motivação, eficácia e mudança de atitude no aluno, que resultam da observação ou da interação social com agentes antropomórficos que são instanciados no papel de modelos sociais. Os investigadores Heeter (1995) e Nam et al (2008) realçam a importância da presença visual do agente, na medida que ele pode reforçar a perceção de que "alguém" é socialmente presente e colaborativo no mesmo espaço, contudo, a utilização de mais do que um agente no mesmo espaço, para uma mesma atividade, pode ter o efeito de "split person" (RUTTKAY, ANDRÉ, \& PELACHAUD, 2010).

De acordo com Baylor \& Kim (2005), será preferível usar múltiplos agentes antropomórficos (figura 1) no mesmo espaço, mas para representarem papéis distintos, como por exemplo o de motivador (fornecendo mensagens para aumentar a confiança), o de perito (fornecendo informação especializada) e o de mentor (combinando o papel de motivador e de perito). 
Figura 1. Quadros de características de função dos Agentes EGA. Fonte: Baylor \& Kim (2005).

\begin{tabular}{|c|c|c|c|}
\hline & Expert & Motivator & Menter \\
\hline Image & & & \\
\hline Animation & Desictic & Emofianal & Deictic a Emotional \\
\hline Voice & Authoritative \& Mosotone & Effusive \& Enthasiastic & Coefident \& Calm \\
\hline Affect & None & $\begin{array}{l}\text { - Acknowledgment } \\
\text { - Confusion } \\
\text { - Exapproval } \\
\text { - Peatement } \\
\text { - Surprise }\end{array}$ & $\begin{array}{l}\text { - Acknowiledgment } \\
\text { - Confusion } \\
\text { - Disapproval } \\
\text { Excitement } \\
\text { - Plcasure } \\
\text { - Surprise }\end{array}$ \\
\hline Script & Information & Encouragement & $\begin{array}{l}\text { Information \& } \\
\text { Encouragement }\end{array}$ \\
\hline
\end{tabular}

Agentes (ECAs) constituem um tipo especial de agentes que podem simular comportamentos verbais e não verbais, a fim de conseguir uma interação mais natural com os humanos a partir de seis emoções básicas: raiva, desgosto, medo, felicidade, tristeza e surpresa, mais uma neutra PERIKOS \& HATZILYGEROUDIS (2014).

Os Agentes (EGAs) fornecem um poderoso meio de comunicação entre humanos e máquinas através da união de gestos, expressão facial/emocional e discurso. A interação face-a-face permite aplicar atos pragmáticos de comunicação, como a ênfase nas frases, representação visual das emoções, ou entonação, conseguida através de canais verbais ou não-verbais, que servem para aumentar a credibilidade, confiança e até mesmo o envolvimento de seus utilizadores REGO (2010).

Figura 2. A faces, representando as seis emoções básicas Ekman \& Friesen (1971).
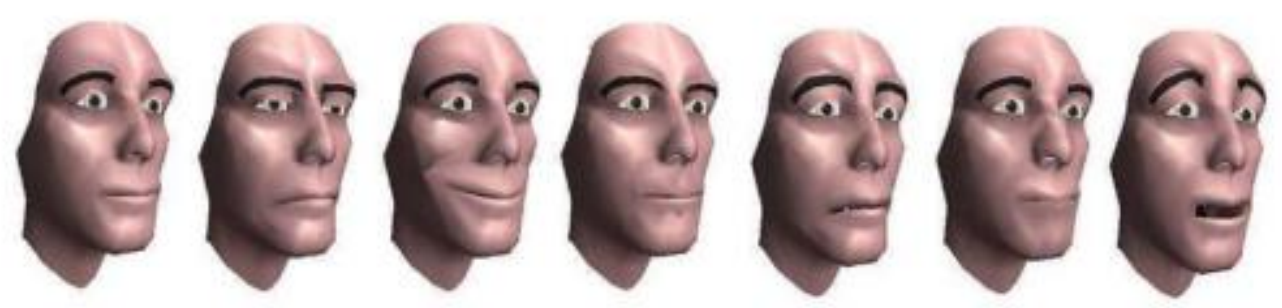

Algumas das expressões faciais de emoção, são universais e iguais para todas as pessoas, independentemente da idade, sexo, raça ou cultura. Estas expressões faciais são a mais rica fonte de informações para comunicação não verbal HEYLEN (N.D.). 
Usando estas funcionalidades, existem alguns projetos, tecnologicamente mais avançados, que tiveram imenso sucesso como por exemplo: XNAgent, é uma plataforma de código aberto para agentes conversacionais incorporados que permite animação dinâmica; Rea (é um agente EGA e uma importante referência no contexto das suas funcionalidades emocionais. É um sistema que suporta uma interação multimodal em tempo real, através de uma interface conversacional baseada em vários protocolos, no sentido de simular uma conversação cara a cara o mais natural possível); Day-today Care (é um sistema que visa auxiliar o idoso nos cuidados necessários ao seu quotidiano para tentar emular o contacto humano que recebem durante todo o dia); e a Farmácia Virtual, um projeto português, promovido pela Faculdade de Ciências da Universidade de Lisboa. A Farmácia Virtual é um projeto gratuito, que tem como objetivo disponibilizar, de forma rápida e precisa, o diagnóstico ou aconselhamento aos utentes em Farmácias. Existem três módulos: Módulo Treino, Avaliação e Backoffice.

Figura 3. Menu principal da Farmácia Virtual.

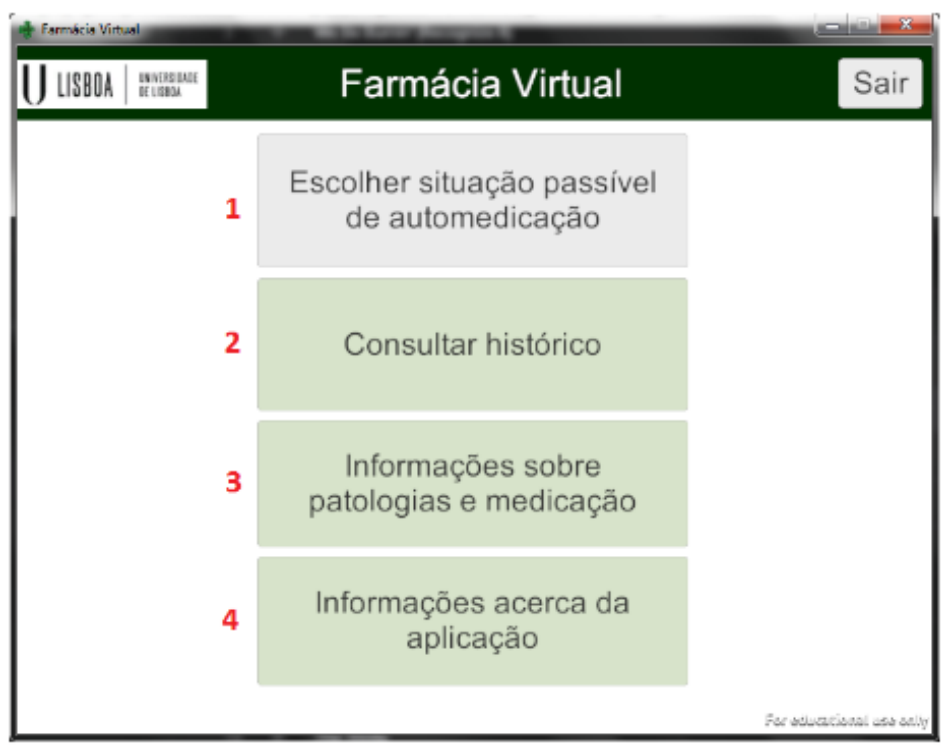

Disponível em: 〈http://virtualpharmacy.fc.ul.pt/Farmacia\%20Virtual\%20-\%20Manual\%20Aplicacao.pdf〉.

\section{BACKGROUND SOBRE AS INVESTIGAÇÕES DESENVOLVIDAS NA ÁREA}

Muitas são as universidades, instituições e investigadores que procuram enriquecer o conhecimento humano com o conhecimento virtual, tarefa que requer um trabalho intenso e uma motivação acrescida, por forma a dotar uma "máquina" com "inteligência", capacidade comunicativa, através da fala (linguagem natural), da imagem ou do som, habilidade de expressar emoções e gestos realistas, interagir com humanos, gerir a perceção não verbal e outros comportamentos e aprendizagens. 
Algumas linhas de investigação que se enquadram nos temas aqui expostos e que são referências para reflexões e aprofundamento das áreas afins estão nas seguintes Universidades: USC - Institute for Creative Technologies da Universidade da Califórnia do Sul (University of Southern California); Universidade de Cambridge e a ArticuLab da Univesidade de Carnegie (Carnegie Mellon University).

O Institute for Creative Technoligies da University of Southern California é uma instituição líder em Inteligência Artificial, tendo 7 grandes áreas de investigação: Ciências de Aprendizagem; Realidade Virtual Médica; Realidades Mistas; Narrativa, Simulação Social, Visão e Gráficos e Humanos Virtuais. A University of Southern California (USC) é líder em Tecnologias Criativas na inteligência artificial, gráficos, realidade virtual, narrativa e tecnologias imersivas para a resolução de problemas, tem nos humanos virtuais uma investigação extensa, nomeadamente em personagens gerados por computador, que proporcionam um meio poderoso para a aprendizagem experiencial, uma vez que usam a linguagem, têm gestos apropriados, mostram emoção e reagem a estímulos verbais e não-verbais. A investigação nesta área é, de longe, considerada a mais avançada. O projeto na área do humano virtual integra aplicações em formação, educação e na promoção da pesquisa em Ciências Sociais, os resultados de uma investigação ativa, constante e sempre inovadora, a (USC) tem produzido ao longo dos anos, resultados de referência em vários projetos, dos quais resultaram alguns protótipos.

O laboratório de computadores usados na investigação na Universidade de Cambridge tem mais de 200 funcionários, desde funcionários académicos, a investigadores associados e a estudantes de doutoramento. A investigação nesta Universidade é feita num vasto leque de áreas da Ciência da Computação, através de Grupos de Pesquisa, em áreas como: Inteligência Artificial; Tecnologia Digital; Gráficos, Programação, lógica (semântica) e Linguagem Natural, onde a investigação é essencialmente em: modelagem computacional das línguas naturais (humanos) e aplicações relacionadas, bem como análise, desenvolvimento e aplicação de ferramentas de raciocínio automatizado.

A Universidade de Carnegie tem uma forte ação na investigação, nomeadamente no campo das interações humanas e a construção de Agentes Virtuais Encapsulados (Embodied Conversational Agents (ECA)) em contextos sociais e culturais. Essa investigação debruça-se, essencialmente, na interação entre os seres humanos e os seus pares virtuais, (ECAs), bem como na forma como os seres humanos interagem uns com os outros através de contextos mediados por computador e investigação. Além disso, há investigações a decorrer que procuram também saber como a tecnologia pode ser usada para iniciativas na área da educação, como por exemplo: a 
melhoria das competências de alfabetização para crianças não nativas na língua Inglesa. Entre os projetos em curso, ou desenvolvidos, são de salientar os projetos: ALEX, RAPT, SARA, SCIPR e o Projecto YAHOO.

O projeto ALEX (linguagem Alex), basicamente, usa a diversidade cultural na sala de aula para comparar resultados entre alunos afroamericanos e euro-americanos, através de uma tecnologia virtual de pares, para compreender o papel do dialeto, as congruências culturais e ajudar os alunos a alcançarem melhores resultados. O RAPT (Rapport-Aligning Peer Tutor) é um projeto que usa Pares Virtuais no suporte à aprendizagem e desenvolvimento que visa analisar a forma como as relações entre alunos são criadas, mantidas e destruídas. O SARA (the Socially Aware Robot Assistant) é uma assistente virtual com consciência social, que depende dela para melhorar as suas funcionalidades. A Sara interage com os utilizadores e personaliza a interação de forma a melhorar o seu desempenho. O Projeto SCIPR (Sensing Curiosity in Play and Responding) tem como base a curiosidade e a motivação dos alunos na aprendizagem da ciência para poderem explorar e produzir conhecimento, assumindo eles o papel de cientistas. Por último, o Projeto YAHOO (CMU InMind) é um Assistente Pessoal, disponível em dispositivos móveis, de criação de relações. Por outras palavras, é um assistente pessoal virtual autónomo que construirá relações duradouras com as pessoas, através da gestão do relacionamento entre elas e ele próprio.

\section{Tendências sobre Tutoria com Agentes Inteligentes na Educação Online}

Em geral, os ambientes virtuais de aprendizagem (AVA) desenvolvidos para a EaD permitem a colaboração e interação, em tempo real, entre professores e alunos, de uma forma remota, de caráter multidimensional, associando simultaneamente diversas tecnologias, como: Internet, webmail, fórum, portefólio, biblioteca, diários, editores colaborativos e chats (Leite, Braglia, \& Pereira, 2011), mas essas não são estimulantes e suficientemente interativas para o tipo de estudante existente atualmente no online.

Kirner (2007) acrescentou ainda que, além da Realidade Virtual permitir um ambiente Colaborativo para a Educação, é também um ambiente multiutilizador, baseado no construcionismo, funcionando na Internet para suporte a diferentes aplicações educacionais, portanto, reforçamos a ideia de que apesar dos avanços tecnológicos terem contribuído consideravelmente para importantes melhorias na $\mathrm{EaD}$, constata-se, pelo elevado nível de desistências nos estabelecimentos de ensino superiores online, que as instituições e os alunos ainda encontram certas adversidades, que afetam a qualidade geral da Educação à Distância. Ultrapassar essas adversidades será um dos maiores desafios que as instituições de EAD enfrentam agora e no futuro. 
O leque de opções que as tecnologias de vanguarda disponibilizam às instituições de $\mathrm{EaD}$ permitem resolver algumas das adversidades encontradas e minimizar outras. As principais tendências, de acordo com a literatura consultada, de alta tecnologia para os próximos anos e que podem mudar significativamente o estado atual da educação à distância, são: a realidade virtual, a realidade aumentada, Inteligência Artificial, Tecnologia Móvel, Gamificação, Social Média, Aprendizagem Digital Data Análise, Computação em Nuvem, Aprendizagem Individualizada e Personalizada e Conteúdos Abertos. Os Agentes Inteligentes fazem parte dessas novas tendências e são realmente caracterizados, como pudemos constatar neste documento, por elementos de potencialização e complementação de algumas das necessidades do trabalho de tutoria, mais trabalhosas e algumas vezes exaustivas para a docência online.

É, contudo, importante que as tecnologias na EaD sejam usadas de acordo com cada situação de uma forma adequada e consideradas como um instrumento pedagógico, que contribuem para melhorar o sucesso dos alunos e das instituições. A partir do contexto e investigações desenvolvidas na área ressalta-se que os Agentes Inteligentes são uma tendência crescente nos espaços formais de aprendizagem (MARCOS, et al.,2018).

\section{CONSIDERAÇÕES FINAIS: ALGUMAS APROXIMAÇÕES}

A evolução tecnológica, particularmente a Inteligência Artificial, tem contribuído significativamente para mudanças relevantes em quase todas as dimensões da nossa sociedade, nomeadamente na Educação e, consequentemente, no Ensino à Distância. Tais mudanças exigem transformações significativas, especialmente no processo de ensino e aprendizagem, de forma a minimizar a incapacidade de os Tutores Virtuais responderem na totalidade às solicitações de suporte por parte dos alunos e, por isso, a necessidade enorme de potenciar as tecnologias existentes, com o objetivo de minorar essa dificuldade.

A caracterização dos agentes inteligentes e a sua importância pedagógica para a tutoria na educação online significa uma outra etapa qualitativa de aprimoramento e ampliação do potencial do eLearning, na personalização dos processos de aprendizagem. O presente artigo caracterizou, em linhas gerais, os principais indicadores das ações e da constituição desses agentes inteligentes, numa tentativa de refletir sobre os diversos conceitos e denominações. De entre o essencial das funções destacámos, de forma breve, os elementos de maior conveniência e importância para o trabalho pedagógico realizado em contextos de educação a distância. Deixaremos para um próximo momento o desenvolvimento de aplicações e resultados pedagógicos desse processo. Como 
resultados, podemos afirmar que a literatura especializada tem destacado a enorme vantagem do uso dos agentes inteligentes no ensino superior online. 


\section{REFERÊNCIAS}

ALVES, D. G. et al. Ambientes virtuais para educação à distância: uma estrutura de classificação e análise de casos IME - Dept de Informática e Ciência da Computação, Universidade do Estado do Rio de Janeiro -UERJ, 2003.

AMARILLA FILHO, P. Educação a distância: uma abordagem metodológica e didática a partir dos ambientes virtuais. Educação Em Revista, 27(2),p. 41-72, 2011.

BARROS, D. M. V.; REIS,V. (2009) A Função tutorial na formação continuada docente. In Revista Iberoamericana de Educación a Distancia, v.1, p.10 - 20.

BARROS, D. M. V; NUNES, J.S Tutoria e docência na educação a distância. UNESP, Material pedagógico em forma de e-book para curso de formação de tutores para os cursos a distância da Especialização em Inclusão via Teleduc, 2011.

BARROS, D. M.V. Estilos de uso do Virtual: estratégias de personalização da aprendizagem In IV Congresso Nacional de Formação de Professores e XIV Congresso Estadual sobre Formação de Educadores - 24 a 26 de setembro de 2018 - Águas de Lindóia, São Paulo.

BAYLOR, A. L. Promoting motivation with virtual agents and avatars: role of visual presence and appearance. Philosophical Transactions of the Royal Society of London. Series B, Biological Sciences, 364(1535), p. 59-65, 2009.

BAYLOR, A. L., \& KIM, Y. Simulating instructional roles through pedagogical agents. In International Journal of Artificial Intelligence in Education, 15(1), p. 95-115, 2005.

BECKER-ASANO, C. WASABI: Affect simulation for agents with believable interactivity, 319, 186. Disponível em: https://becker-asano.de/Becker-Asano_WASABI_Thesis.pdf. Acesso em: 30 setembro 2018.

BELLONI, M. L. Educação a Distância. Campinas: Autores Associados, 2008.

CARNEGIE MELLON UNIVERSITY, Articulab, Disponível em: http://articulab.hcii.cs.cmu.edu/projects/ Acesso em: 30 maio 2018.

CASSEl, J., Sullivan, J., PREVOST, S., CHURCHILl, E., TROITZSCH, H., CASSEL, J., CHURCHILL, E. Embodied Conversational Agents In Embodied Conversational Agents, 40(1), 26-36. 2000.

CEJUDO, M. El tutor en e-learning: aspectos a tener en cuenta. In Revista Electrónica de Tecnologia Educativa, 20, 1-24, 2006. Disponível em: <http://www.quadernsdigitals.net/datos/hemeroteca/r_11/nr_180/a_10016/10016.pdf>. Acesso em: 30 setembro 2018.

CHAO,C. ET AL. An Affective Learning Interface with an Interactive Animated Agent. In IEEE Fourth International Conference On Digital Game And Intelligent Toy Enhanced Learning.Ieee, p.221-225, 2012.

CINTO, T., LEITE, H., PEIXOTO, C., \& ARANTES, D. Eixo 4 - tecnologia e projetos inovadores para a educação a distância. In The use of Computational Avatars as Tools for e-learning teaching, p. 960-1035, 2013.Communication in the age of virtual reality. Hillsdale, NJ: Lawrence Erlbaum Associates.

EKMAN, P., \& FRIESEN, W. V. Constants across cultures in the face and emotion. In Journal of 27 Personality and Social Psychology. 1971.

GOMES, C. Chatbot: entenda tudo sobre o assunto, 2017. Disponível em: <http://blog.simply.com.br/chatbot/> . Acesso em: 30 maio 2018.

GONÇAlves, H. O Perfil do Professor/Tutor em Cursos Online. Dissertação de Mestrado. Universidade Aberta, Lisboa, 2008.

HEETER, C. Communication research on consumer VR. F. Biocca \& M. Levy (Eds.), 1995.

HEYLEN, D. (n.d.). Facial Expressions for Conversational Agents. University of Twente - Netherlands.

HSU, Y. Affective Interfaces of Embodied Conversational Agents. In International Journal of Affective Engineering, 12(2), p.71-78, 2012.

JOHNSON, L., ADAMS BECKER, S., CUMMINS, M., ESTRADA, V., \& FREEMAN, A., AND HALL, C. Horizon Report: 2016 Higher Education Edition. Reading, 2015.

LIN,H.,TSAI,I. An Ontology-Based Affective Tutoring System on Digital Arts. In IEEE Workshop on Affective Computational Intelligence, 2011. 
MARCOS A.; CLÁUDIO A.P.; MARTINHO,C.; BARROS,D.M.V.; CARVALHO,E.; CARMO,M.B:; SÓNIA SEIXAS,S. Virtual Tutoring, In 9th annual International Conference of Education, Research and Innovation, Barcelona In Costa MF, Dorrio BV, Fernandez-Novell JM (Eds.) International Conference of Education,p. 56-61, 2018.

MACHADO, L. D.; MACHADO, E. C. O papel da tutoria em ambientes de ead. In Formação de Profissionais para Educação a Distância - Educação Universitária, abril 2004.

MAHMOUD, M., \& ROBINSON, P. Interpreting hand-over-face gestures. Lecture Notes in Computer Science (Including Subseries Lecture Notes in Artificial Intelligence and Lecture Notes in Bioinformatics), 6975 LNCS(PART 2),p. 248-255, 2011.

MARSI, E., ROODEN,F. Expressing Uncertainty with a Talking Head in a Multimodal Question-Answering System, Tilburg University, Netherlands, 2007.

MELO, C. M. Agentes Antropomórficos, IST, AASM, 2007.

MORGADO, L. O papel do professor em contextos de ensino "online": problemas e virtualidades In Discursos: perspectivas em educação. Lisboa. 3 , no especial, p.125-138, 2001.Diponível em: http://hdl.handle.net/10400.2/1743

MORGADO, L. Ensino Online: Contextos e Interacões, Tese de Doutoramento, Lisboa: Universidade Aberta, 2003.

MORGADO, L. Tutoria e Tutor em Educação a Distância: Retratos do Presente versus Visões para o Futuro, Educação a Distância em Foco, 5 (1), p.64-87, 2015.

MULDOWNEY, O. Chatbots: an introduction and easy guide to making your own. Dublin, Ireland: Curses \& Magic, 2017.

NASCIMENTO, L. ET AL. Tutoria e Tutor em Educação a Distância: Retratos do Presente versus Visões para o Futuro, In Educação a Distância em Foco, 5 (1), p.64-87, 2015.

NAM, C. ET AL. The roles of sensory modalities in collaborative virtual environment. Computers in Human Behavior, 24(4), p.1404-1417, 2008.

PERIKOS, I., \& HATZILYGEROUDIS, L. Embodied Coversational Agents: a methodology for Learning to Express Facial Emotions. University of Patras, 2014.

REGO, S. Fala Comigo: Agente Virtual Conversacional e Emocional. Dissertação para obtenção do grau de Mestre em Engenharia Informática e de Computadores do Instituto Superior Técnico - Lisboa, Portugal, 2010.

RICKEL, J., INFORMATION, U. S. C., ANDRE’, E., BADLER, N., \& CASSELL, J. Virtual Humans: Some Assembly Required, 2002a.

RICKEL, J., INFORMATION, U. S. C., ANDRE', E., BADLER, N., \& CASSELL, J. Creating Interactive Virtual Humans: SomeAssembly Required, 2002b.

RUTTKAY, Z., ANDRE, E., L., J., \& PELACHAUD, C. Dagstuhl Seminar. It - Information Technology, 46(52004), p.1-16. 2010.

SANGRÁ, A., VLACHOPOULOS, D., CABRERA, N., \& BRAVO, S. Hacia una definición inclusiva del elearning, 2011. Disponível em: http://openaccess.uoc.edu/webapps/o2/handle/10609/10541\nhttp://openaccess.uoc. edu/ webapps/o2/bitstream/10609/10541/6/inf_ed_cast.pdf. Acesso em: 30 maio 2018.

SCHAPPO, V. Chatbot: o que é, quais são as suas as vantagens e como usar na sua empresa. Blog de Marketing Digital de Resultados, 2017. Disponível em: <https://resultadosdigitais.com.br/blog/chatbot/>. Acesso em: 2 julho 2018.

SRDANOVIC, B. Chatbots In Education: applications of chatbot technologies. eLearning Trends, 2017. Disponível em: <https://elearningindustry.com/chatbots-in-education-applications-chatbot-technologies>.

ZUMSTEIN, D.; HUNDERTMARK, S. Chatbots - an interactive technology for personalized communication, transactions and services. In IADIS International Journal on WWW/Internet, v. 15, n. 1, 2017. 


\section{RESUMO}

A evolução tecnológica, particularmente a Inteligência Artificial, tem contribuído significativamente para mudanças relevantes em quase todas as dimensões da nossa sociedade, nomeadamente na Educação e, consequentemente, no Ensino à Distância. Tais mudanças exigem transformações significativas, especialmente no processo de ensino e aprendizagem, de forma a minimizar a incapacidade dos docentes e tutores responderem na totalidade às solicitações de suporte por parte dos alunos e, por isso, a enorme necessidade de potenciar as tecnologias existentes, com o objetivo de minorar essa dificuldade. Este artigo tem como objetivo caracterizar os agentes inteligentes e a sua importância pedagógica para a tutoria na educação online. A metodologia utilizada baseou-se no método dedutivo, resultante do processo de pesquisa bibliográfica e documental, em consonância com a investigação de doutoramento em curso sobre a temática dos tutores inteligentes. Como resultados, a literatura especializada tem destacado a enorme vantagem da utilização dos agentes inteligentes no ensino superior online.

Palavras-chave: Tutoria Virtual, Agentes Inteligentes, Educação Online

\section{TUTORING WITH INTELLIGENT AGENTS IN ONLINE EDUCATION}

\section{ABSTRACT}

Technological evolution, particularly Artificial Intelligence, has contributed to changes in almost all dimensions of our society, in education and in Distance Learning. Such changes require significant transformations in the teaching and learning process in order to minimize the inability of Virtual Tutors to respond to students' requests for support and, therefore, the need to improve existing technologies, with the aim of reducing this difficulty. This article aims to characterize intelligent agents and their pedagogical importance for tutoring in online education. The methodology used was based on the deductive method, resulting from the bibliographic and documentary research process, in consonance with the ongoing doctoral research on the subject of virtual tutors. As a result, the literature has highlighted the enormous advantage of using intelligent agents in online higher education.

Keywords: Virtual Tutoring, Smart Agents, Online Education

\section{TUTORÍA CON AGENTES INTELIGENTES EN LA EDUCACIÓN ONLINE}

\section{RESUMEN}

La evolución tecnológica, particularmente la Inteligencia Artificial, ha contribuido a los cambios en casi todas las dimensiones de nuestra sociedad, en la educación y en la Enseñanza a Distancia. Estos cambios requieren transformaciones significativas en el proceso de enseñanza y aprendizaje para minimizar la incapacidad de los Tutores Virtuales para responder a las solicitudes de apoyo por parte de los alumnos y por lo tanto la necesidad de mejorar las tecnologías existentes con el objetivo de aliviar esa dificultad. Este artículo tiene como objetivo caracterizar a los agentes inteligentes y su importancia pedagógica para la tutoría en la educación online. La metodología utilizada fue basada en el método deductivo, resultante del proceso de investigación bibliográfica y documental, en consonancia con la investigación de doctorado en curso sobre la temática de los tutores virtuales. Como resultados, la literatura especializada ha destacado la enorme ventaja del uso de los agentes inteligentes en la enseñanza superior en línea.

Palabras clave: Tutoriales virtuales, Agentes inteligentes, Educación en línea

Submetido em Julho de 2019 Aprovado em Outubro de 2019 